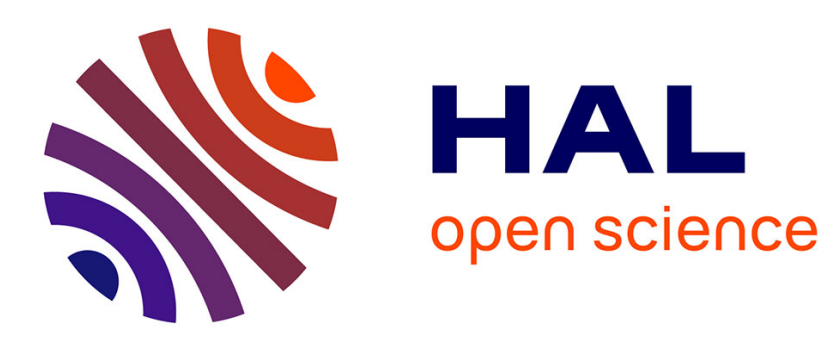

\title{
How Does Asymmetric Information Create Market Incompleteness?
}

\author{
Anne Eyraud-Loisel
}

\section{To cite this version:}

Anne Eyraud-Loisel. How Does Asymmetric Information Create Market Incompleteness?. Methodology and Computing in Applied Probability, In press. hal-01998386

\section{HAL Id: hal-01998386 \\ https://hal.science/hal-01998386}

Submitted on 29 Jan 2019

HAL is a multi-disciplinary open access archive for the deposit and dissemination of scientific research documents, whether they are published or not. The documents may come from teaching and research institutions in France or abroad, or from public or private research centers.
L'archive ouverte pluridisciplinaire HAL, est destinée au dépôt et à la diffusion de documents scientifiques de niveau recherche, publiés ou non, émanant des établissements d'enseignement et de recherche français ou étrangers, des laboratoires publics ou privés. 
Noname manuscript No.

(will be inserted by the editor)

\title{
How Does Asymmetric Information Create Market Incompleteness?
}

\section{Anne EYRAUD-LOISEL}

Received: date / Accepted: date

\begin{abstract}
The aim of this work is to show that incompleteness is due in general not only to a lack of assets, but also to a lack of information. In this paper we present a simple influence model where the influencial agent has access to additional information. This leads to the construction of two models, a complete model and an incomplete model where the only difference is a difference of information. This leads to a simple model of incomplete market where the number of assets is not the cause of incompleteness: incomplete information is the explanation.
\end{abstract}

Keywords Information · asymmetric information · option pricing · martingales · insider trading $\cdot$ complete market $\cdot$ incomplete market

AMS Classification (2000): 60H10, 60G44, 60H07, 60J75, 91G20, 91B70, 93E11.

JEL Classification: C60,G11,G14.

\section{Introduction}

In economics, incomplete markets are viewed as markets in which the number of Arrow-Debreu securities is less than the number of states of nature [2]. In finance, complete markets are markets in which any contingent claim is attainable, which means that it can be written by means of admissible strategy based on existing assets in the market, and incomplete markets are non-complete markets. In both definitions, one generally assumes that we are in presence of perfect information.

A. Eyraud-Loisel

Université de Lyon, Université Lyon 1, ISFA, Laboratoire SAF

50 avenue Tony Garnier 69007 LYON

Tel +33-437-28-74-35 E-mail: anne.eyraud@univ-lyon1.fr 
The most well-known cause of the incompleteness of markets is the lack of assets: despite the high number of claims traded routinely against many states (such as insurance policies, futures, options), the set of outcomes is always greater than the set of claims, and financial and insurance markets remain incomplete. Other sources of incompleteness of markets are market frictions and ambiguity (see for example [15]). According to Staum, market frictions, such as transaction costs and constraints on portfolios, may cause incompleteness. He also points out that ambiguity may be a source of effective incompleteness, when agents ignore the true stochastic model for market prices. These phenomena are particularly enlightened when looking at incompleteness in finite market models, and in equilibrium problems. In discrete market models, with a finite number of state of nature, it is quite easy to link it with a lack of assets. For a more detailed study, the reader may consult Duffie et al. [3] in which the link between frictions and liquidity in OTC markets and valuation is addressed.

Nevertheless another less known source of incompleteness of markets may be asymmetric information. Instead of explaining the incompleteness of the market by missing assets, it is explained by missing information instead. In insurance, market incompleteness often combines lack of replicating assets and asymmetry of information.

Notice that in this paper, we have chosen to study continuous-time market models, and that we will not deal with equilibrium issues. The main point of this study is to take a given price market model, and to discuss whether it can be incomplete or not, depending on the information possessed by the agents. For more information on equilibrium studies with asymmetrical information, the reader can refer to [13] for example.

The aim of the present article is to highlight the role of asymmetric information on the incompleteness of markets. The main point is to construct a market where all the incompleteness is created only by a lack of information, and by that to isolate the asymmetry of information effect on the incompleteness of markets. In the model that is presented, the incomplete market is completed when adding the information.

There actually exist differences between agents' information on the market. The available information is not identical if the investor is an owner, a manager, or a simple agent. Different kinds of investors have access to different kinds of information. How does investors' information influence their strategies when investing on markets? This question has already been studied in the litterature: from an optimization point of view at first (see Grorud [9], Grorud and Pontier $[10,11,12]$ ), and from a hedging point of view more recently (see Eyraud-Loisel $[4,5,6])$.

In [4], a simple Black-Scholes style model with an informed investor is studied, proving that under reasonable assumptions, no additional hedging strategy is provided by a strong initial information on future prices, when the the information concerns a time horizon larger than the time horizon of the hedging problem. The main hypothesis is that the agent is supposed to be a small investor: the standard agent invests without any influence on market 
prices. A small investor observes market prices, and her investment choices do not have any effect on the dynamics of prices.

In [5], the model is improved: the main (and informed) investor is supposed to be influential. It means that the investor is supposed to have an influence on the dynamics of prices: she can either be a large investor, which means that she invests such a large amount of money in the asset that her investment may modify the equilibrium problem leading to the price dynamics, or an influent investor, which means that her strategy on the market may be followed, or known, and may have an influence on the equilibrium problem also, by driving other investors for example (such as charter's phenomena). It seems reasonable to suppose that if the investor has access to a privileged information, then she may be such an influential agent. Once again, the equilibrium process that leads to the influence of the agent on the market will not be addressed, but the influence of the agent will be taken as an assumption.

Last, in [6], the model is studied from the point of view of a non informed investor trying to invest on such an influenced market model. For a deep mathematical presentation of this modeling, the reader can refer to the papers cited before. In the present work, we quickly present the different models in order to point out their interest in terms of financial markets mechanisms, and to give a new perspective of to market incompleteness. Despite the completeness of the influenced informed market, the non-informed market is incomplete: the common agent does not have access to the entire information driving the prices process, and this generates the incompleteness of the market.

The aim of the present article is to present this incomplete market, where the incompleteness of the market is due to the presence of an influent informed investor, and to give an explanation of such an incomplete market by means of missing information.

The article is organized as follows: Section 2 presents the complete market model. Section 3 is devoted to the presentation of the incomplete market derived from the previous complete market, when retrieving a part of the information driving the complete market. Section 4 presents a measure of the incompleteness by means of measuring the lack of information that leads to the incompleteness of the market. Section 5 presents the implications of such highlighting of the role of asymmetric information on incompleteness of markets.

\section{The Complete Market Model}

In [4], it has been proven that in a small investor framework, if the market is complete, no additional hedging strategy is created when accessing to an additional information. This is not what we are looking for here. So we suppose that on a simple complete Black-Scholes model, an agent has some influence on the asset prices. The influent informed agent is also supposed to possess an additional information, and is also supposed to influence the market. This is a natural extension of the previous work of Eyraud-Loisel (2005) [4], where 
the informed agent is supposed to be a small investor, who does not influence asset prices.

Let us take a simple model (for instance the Black Scholes model), where the price process is written as a stochastic differential equation, under a probability space $(\Omega, P)$ :

$$
P_{t}=P_{0}+\int_{0}^{t} b\left(s, P_{s}\right) d s+\int_{0}^{t} \sigma\left(s, P_{s}\right) d W_{s},
$$

where $W$ is a standard Brownian Motion, $b$ and $\sigma$ having standard hypotheses to ensure completeness of this market.

Let us add the possibility that $X$ and $\pi$ the wealth and portfolio of a large or influential agent may influence the standard drift $b$ and volatility $\sigma$ of prices. The risky asset price $P_{t}$ is then supposed to be driven by the extended following stochastic differential equation:

$$
P_{t}=P_{0}+\int_{0}^{t} b\left(s, P_{s}, X_{s}, \pi_{s}\right) d s+\int_{0}^{t} \sigma\left(s, P_{s}, X_{s}, \pi_{s}\right) d W_{s} .
$$

The market is complete without this new influence hypothesis, but what about the completeness of the market when this influence is added ?

The influence hypothesis may be expained as follows: the informed trader may influence asset prices dynamics, both by being a "Large investor" (her wealth $X$ may influence drift $b$ and volatility $\sigma$ of price dynamics), and by being an "influential investor": her investment strategy $\pi$ may influence drift $b$ and volatility $\sigma$ of price dynamics.

The chosen model is the same as in [5], where the market price process may be influenced by the informed agent. It is more realistic to consider large traders in the market: investors who have access to priviledged information are often big investors, who may influence the evolution of asset prices. This influence can either be by means of their large investment depth, or by their notoriety, when a charter phenomenon appears. Thus, asset prices may be influenced by certain "big agents" on the market, and it is quite natural to suppose that such large agents may have more easily access to additional information on the market.

The additional information is supposed to be an information on the price at a certain future time horizon (larger than the horizon of the considered price model). Mathematically speaking, information is a strong initial information, modeled by an initial enlargement of the Brownian filtration, as developed for insider trader modeling in Grorud and Pontier [10], or Amendinger [1], satisfying the so-called "Hypothesis $\left(\mathbf{H}_{\mathbf{3}}\right)$ ", where martingales under the small filtration remain martingales under the larger filtration and a change of probability. This influenced market has been already studied in [5] from the influent informed agent's point of view, by solving the Forward-Backward Stochastic Differential Equation (FBSDE) that appears when modeling the hedging problem of the large trader, under the enlarged filtration induced by the additional information. The standard Brownian filtration will be denoted by $\mathcal{F}$, modeling normal standard information, accessible to any standard investor, and 
enlarged filtration containing additional information will be denoted by $\mathcal{Y}$, only accessible to the informed investor. So as $(\Omega, P)$ denotes the probability space of the model, $(\Omega, \mathcal{F}, P)$ denotes the filtered Brownian probability space, and $(\Omega, \mathcal{Y}, P)$ the filtered enlarged space.

The problem leads to a coupling between the forward equation of prices and the backward equation of wealth, which fits the framework of the standard following generalized Forward-Backward Stochastic Differential Equation (FBSDE) (where $Z$ is simply a generalization of previous $\pi$ ):

$$
\left\{\begin{array}{l}
P_{t}=P_{0}+\int_{0}^{t} b\left(s, P_{s}, X_{s}, Z_{s}\right) d s+\int_{0}^{t} \sigma\left(s, P_{s}, X_{s}, Z_{s}\right) d W_{s} \\
X_{t}=\xi-\int_{t}^{T} f\left(s, P_{s}, X_{s}, Z_{s}\right) d s-\int_{t}^{T} Z_{s} d W_{s} .
\end{array}\right.
$$

Under Lipschitz, linear growth and integrability hypotheses on $b, \sigma, f$ and $\xi$ (cf Pardoux-Tang [14]), as well as additional integrability hypotheses under an equivalent probability measure $Q$ (the probability measure under which Hypothesis $\left(\mathbf{H}_{\mathbf{3}}\right)$ holds), existence and uniqueness can be proved. There are three cases where results are obtained.

- Weak influence: $b$ and $\sigma$ weakly depend on $X$ and $Z$.

- The agent wants to hedge a finite value a.s.: $\xi$ does not depend on price $P$.

- The portfolio does not influence volatility of prices: $\sigma$ is independent of $Z$.

The main proven result (Theorem 3.1 in [5]) may be stated as follows.

Proposition 2.1 Under hypothesis $\left(\mathbf{H}_{3}\right)$ on the private information, for any functions $f, b, \sigma$ satisfying standard Lipschitz, linear growth and integrability hypotheses and at least one of the 3 influence cases, there exists a unique solution of the FBSDE in the enlarged space $(\Omega, \mathcal{Y}, Q)$ under the change of probability $Q$.

Refer to [5] for more details on the hypotheses.

The important point for our purpose here is the financial interpretation of this result: the influent agent has a unique admissible hedging strategy. The strategy is adapted to $\mathcal{Y}$, the enlarged filtration containing the entire information. How can we compare this strategy with the strategy of a non informed trader investing on this market?

\section{Incompleteness of the market without the entire information}

The market with the entire information possessed by the informed agent is complete. But the market without the entire information is incomplete, whereas the number of assets is unchanged, and no friction is added. Moreover, it is possible to complete the market by simply adding the knowledge of the private information that is missing to the average agent (small investor investing on the market, but who has only access to the information generated by prices, and not to the private information). The study of this incomplete market from the mathematical point of view was the aim of [6], which was 
devoted to the study of the non-informed agent's strategy in this influenced market.

To sum up the results, a non informed agent investing on the influenced market has the information $\tilde{\mathcal{F}}$ filtration generated by prices, which satisfies

$$
\mathcal{F} \subset \tilde{\mathcal{F}} \subset \mathcal{Y} .
$$

The common agent does not know the private information that is driving the market, but she observes prices on the market. Prices are partly driven by the private information, because of the influence of the informed agent. But she has no access to entire information driving the market. She is not able to distinguish between the part of information that is revealed on the market and the part that comes directly from the Brownian standard natural filtration (basic information of prices). So the information she has access to is greater than the natural Brownian filtration (as some of the private information is somehow revealed by the market) but less than the entire information. Mathematically speaking, under such a filtration (set of information), there is no Martingale Representation Theorem under $\tilde{\mathcal{F}}$, which implies that the studied market is an Incomplete market for the non-informed trader. This also means that no general solution of a hedging Backward Stochastic Differential Equation under filtration $\tilde{\mathcal{F}}$ can be found.

Different methods may be used in order to solve the hedging problem for any contingent claim under such a filtration: we choose the Kunita-Watanabe (K-W) Decomposition in order to replace the lack of exact martingale representation theorem, as well as methods of quadratic hedging in incomplete markets as in Follmer-Schweizer [7]. We present here the main results of [6].

Let us denote by $\mathcal{Q}$ (respectively $\mathcal{Q}_{N}$ ) the set of $\mathcal{Y}$ (respectively $\tilde{\mathcal{F}}$ )martingale measures (risk-neutral probabilities for the informed trader (respectively non informed trader).

Definition 3.1 If $N, M \in \mathcal{M}^{2}(\mathcal{G}, \mathbb{P})$ the space of square integrable $(\mathcal{G}, \mathbb{P})$ martingales, then the unique Kunita-Watanabe decomposition of $N$ w.r.t. $M$ is

$$
N_{t}=N_{0}+\int_{0}^{t} \theta_{u} d M_{u}+L_{t}, \mathbb{P}-p . s .
$$

where $\theta \in L^{2}(\Omega)$ is square integrable, and where $L \in \mathcal{M}_{0}^{2}(\mathcal{G}, \mathbb{P})$ is a square integrable $(\mathcal{G}, \mathbb{P})$-martingale with 0 expectation, and is orthogonal to $M$ in $(\mathcal{G}, \mathbb{P})$.

Let $\tilde{Q} \in \mathcal{Q}$, and $\xi \in \mathcal{L}^{2}(\Omega, \tilde{\mathcal{F}}, \tilde{Q})$ the space of all square-integrable $(\mathcal{F}, \tilde{Q})$ adapted processes. The martingale representation Theorem under filtration $\mathcal{Y}$ gives

$$
\xi=E_{\tilde{Q}}(\xi \mid \sigma(L))+\int_{0}^{T} \phi_{s}^{L} d P_{s},
$$

where we can also prove that $E_{\tilde{Q}}(\xi \mid \sigma(L))$ and $\phi_{s}^{L}$ do not depend on the choice of $\tilde{Q} \in \mathcal{Q}$. 
Under any $\tilde{Q} \in \mathcal{Q}_{N}$, we have the $\mathrm{K}-\mathrm{W}$ decomposition of $\xi$ w.r.t. $(\tilde{\mathcal{F}}, \tilde{Q})$ and the martingale $P$ :

$$
V_{t}:=E_{\tilde{Q}}\left(\xi \mid \tilde{\mathcal{F}}_{t}\right)=E_{\tilde{Q}}(\xi)+\int_{0}^{t} \phi_{s}^{\tilde{Q}} d P_{s}+L_{t}^{\tilde{Q}} .
$$

Proposition 3.2 (Filtering Result from [6]) Under any $\tilde{Q} \in \mathcal{Q}$, the integrand of this decomposition may be written

$$
\phi_{s}^{\tilde{Q}}=E_{\tilde{Q}}\left(\phi_{s}^{L} \mid \tilde{\mathcal{F}}_{s}\right) .
$$

\section{Measuring lack of information}

The lack of information is crucial in this precise incomplete market: it is the only source of incompleteness. It highlights the role of asymmetric information in the incompleteness of markets. When agents do not have access to the entire information driving the market, the market is incomplete. If all the information is accessible, then the market is complete. An important question is how to measure this lack of information. It could give a way to measure the incompleteness of this market, which is entirely linked to the lack of information.

A deep study and discussion on the best way to chose a measure for the lack of information could be an interesting further work. In general, one could use criteria like Kullback-Leibler divergence, entropy minimization or measure of lack of information focusing on certain subsets of sample paths, corresponding to events triggering particular consequences for the financial products considered. In our context, the approach of Föllmer and Sondermann [8] is the most commonly used and relevant one. Here we choose this standard approach to point out the importance of giving a measure, and to show that it is possible to obtain an expression of the quadratic residual risk. We follow the standard method introduced by Föllmer and Schweizer [7] on the quadratic risk (see also Föllmer and Sondermann [8]), we study the variance of $L_{T}^{\tilde{Q}}$, and we obtain an expression of the quadratic residual risk which measures the risk taken by an agent who does not know information $L$.

For a probability measure $\tilde{Q} \in \mathcal{Q}_{N} \backslash \mathcal{Q}$, which is an equivalent martingale measure for the non informed trader, but not for the informed trader, we measure incompleteness thanks to the following expression:

$$
\operatorname{Var}_{\tilde{Q}}\left(L_{T}^{\tilde{Q}}\right)=E_{\tilde{Q}}\left(\left(\xi-E_{\tilde{Q}}(\xi)\right)^{2}\right)-E_{\tilde{Q}}\left(\left(\int_{0}^{T} \phi_{s}^{\tilde{Q}} d P_{s}\right)^{2}\right) .
$$

For a probability measure $\mathbb{Q}^{*} \in \mathcal{Q}$, we obtain a more simple expression:

$$
\operatorname{Var}_{\mathbb{Q}^{*}}\left(L_{T}^{\mathbb{Q}^{*}}\right)=E_{\mathbb{Q}^{*}}\left(E_{\mathbb{Q}^{*}}\left[E_{\mathbb{Q}^{*}}(\xi \mid \sigma(L)) \mid \tilde{\mathcal{F}}_{T}\right]^{2}\right)-E_{\mathbb{Q}^{*}}(\xi)^{2} .
$$


A minimum risk exists, and it corresponds to the minimum risk linked to "un-information". The agent does not have all the information driving the market. In order to measure the lack of information, we consider the hedging strategy of the non informed agent that minimizes the risk under a given equivalent martingale measure. It corresponds to the conditional expectation of the insider's hedging strategy, given the information $\mathcal{F}^{P}$ of the non informed agent. This gives a measure of the lack of information. However, this provides only a measure of the lack of information under a given probability measure, which is arbitrary. The evaluated risk is the risk of the model under the chosen risk-neutral probability measure. It is not the internal model risk measure. In particular, the historical probability measure is not taken into account. A way to obtain a measure of the intrinsic lack of information of the market in this model could be to choose the risk-neutral measure that minimizes this quantity.

\section{Conclusion}

Such model is not standard, and even if it is more difficult to deal with the problem when no representation property exists, it gives a simple model where all the incompleteness of the market is explained by a lack of information. We isolated the asymmetric information effect on incomplete markets, as only asymmetry of information makes the market incomplete.

The main conclusion is that it is important to consider both the number of assets and the availability of information when studying the completeness or incompleteness of a market.

An interesting point would be to study this incomplete market more deeply, and especially try to express the link between the filtration generated by prices and the lack of information. One could also study which exact part of information is transferred to the market. The private information has been proved to be enough to complete the market, by construction, but one can wonder what kind of information is sufficient to complete this incomplete market, due to the lack of information. Another unsolved problem corresponds tothe choice of the best risk-neutral probability: what is the best way to measure the internal risk of investing in a market with a lack of information?

It not necessarily relevant to focus on this phenomenon when investing in very liquid markets, where additional information like insider information is prhaps not so common. However, when using standard financial pricing or hedging techniques to less liquid markets, or markets with more asymmetric information problems (insurance markets, commodity markets, wheather derivatives markets,...), it turns out to be very important to focus on any possible source of incompleteness of markets, especially the lack of information. 


\section{References}

1. J. Amendinger. Initial enlargement of filtrations and additionnal information of financial markets. Doctoral Thesis, 1999.

2. K. Arrow. The role of securities in the optimal allocation of risk bearing. Review of Economic Studies, (31):91-96, 1964.

3. D. Duffie, N. Gârleanu, and L.H. Pedersen. Valuation in over-the-counter markets. Working Paper.

4. A. Eyraud-Loisel. Backward stochastic differential equations with enlarged filtration. option hedging of an insider trader in a financial market with jumps. Stochastic Processes and their Applications, 115(11):1745-1763, 2005.

5. Anne Eyraud-Loisel. Option hedging by an influential informed investor. Appl. Stoch. Models Bus. Ind., 27(6):707-722, 2011.

6. Anne Eyraud-Loisel. Quadratic hedging in an incomplete market derived by an influential informed investor. Stochastics, 85(3):412-430, 2013.

7. H. Föllmer and M. Schweizer. Hedging of contingent claims under incomplete information. In Applied stochastic analysis (London, 1989), volume 5 of Stochastics Monogr., pages 389-414. Gordon and Breach, New York, 1991.

8. H. Föllmer and D. Sondermann. Hedging of nonredundant contingent claims. In Contributions to mathematical economics, pages 205-223. North-Holland, Amsterdam, 1986.

9. A. Grorud. Asymetric information in a financial market with jumps. Int. Journal of Theor. and App. Fin., 3(4):641-659, 2000.

10. A. Grorud and M. Pontier. Insider trading in a continuous time market model. Int. Journal of Theor. and App. Fin., 1(3):331-347, 1998.

11. A. Grorud and M. Pontier. Probabilités neutres au risque et asymétrie d'information. C. R. Acad. Sci. Paris Sér. I Math., 329(1):1009-1014, 1999.

12. A. Grorud and M. Pontier. Asymetrical information and incomplete markets. Int. Journal of Theor. and App. Fin., 4(2):285-302, 2001.

13. C. Hillairet. Existence of an equilibrium with discontinuous prices, asymmetric information, and nontrivial initial $\sigma$-fields. Math. Finance, 15(1):99-117, 2005.

14. E. Pardoux and S. Tang. Forward-backward stochastic differential equations and quasilinear parabolic PDEs. Probab. Theory Related Fields, 114(2):123-150, 1999.

15. J. Staum. Incomplete markets. In Handbooks in Operations Research and Management Science, volume 15 of Financial Engineering. Birge J.R., Linetsky V., editors., pages 511-563. 2008. 